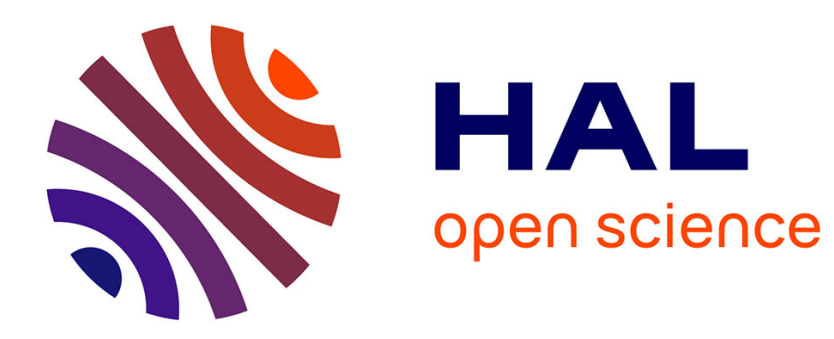

\title{
Sur la distribution des intervalles d'émission des particules $\alpha$ du polonium
}

\author{
Pierre Curie
}

\section{To cite this version:}

Pierre Curie. Sur la distribution des intervalles d'émission des particules $\alpha$ du polonium. Radium (Paris), 1911, 8 (10), pp.354-356. 10.1051/radium:01911008010035401 . jpa-00242490

\section{HAL Id: jpa-00242490 https://hal.science/jpa-00242490}

Submitted on 1 Jan 1911

HAL is a multi-disciplinary open access archive for the deposit and dissemination of scientific research documents, whether they are published or not. The documents may come from teaching and research institutions in France or abroad, or from public or private research centers.
L'archive ouverte pluridisciplinaire HAL, est destinée au dépôt et à la diffusion de documents scientifiques de niveau recherche, publiés ou non, émanant des établissements d'enseignement et de recherche français ou étrangers, des laboratoires publics ou privés. 


\section{Sur la distribution des intervalles \\ d'émission des particules a du polonium \\ Par $M^{\text {me }}$ P. CURIE \\ [Faculté des Sciences de Paris.]}

Dans des travaux récemment publiés, MM. Ruther- d'autre part, ont étudié la distribution dans le temps ford et Geiger, d'une part, MI. Marsden et Barratt, de l'émission des particules $\alpha$ du polonium $\mathrm{cl}$ de 
l'uranium. Les résultals obtenus sont conformes à la formule théorique, indiquée par M. Bateman ${ }^{1}$. Les expériences étaient faites par la méthode des scintillations, chaque scintillation étant enregistrée par l'observateur au moyen d'un dispositif convenable.

Il y a quelques mois j’ai eu également l'occasion d'étudier la loi de l'émission des particules $\propto$ du polonium. Cette étude qui fait partic d'un travail plus étendu ne devait être publiée que plus tard. Cependant, eu égard aux publications précédemment citćes, je crois utile d'indiquer dès à présent les résultats qui confirment ceux obtenus par les autres observateurs.

La substance radioactive étudiće est le polonium. Depuis deux ans, je poursuis, en collaboration avec M. Debierne, un travail ayant pour but la préparation de polonium en quantité aussi grande que possible et à l'état de concentration aussi avancée que possible. Ce travail a déjà permis de mettre en évidence quelques raies nouvelles, appartenant selon toute vraisemblance au polonium, et de prouver la production d'hélium par le polonium. Nous nous proposons de plus : $1^{\circ}$ De fixer la nature du produit de désagrégation du polonium (plomb?) ; $2^{\circ}$ d'obtenir, par la mesure du volume de gaz hélium formé et par la numération directe des particules $\alpha$ qui contribuent à la formation de ce gaz, la valeur de la constante d'Avogadro, ou nombre de molécules contenues dans une moléculegramme.

Les expériences de numération des parlicules $\alpha$, entreprises dans le but indiqué ci-dessus, sont poursuivies à la fois par la méthode des scintillations et par la méthode électrométrique de M. Ruth rorford, dans laquelle l'entrée de chaque particule dans une chambre d'ionisation se traduit par une impulsion communiquée à un électromètre, l'effet ionisant de la particule étant considérablement augmenté gràce à l'utilisation du phénomène d'ionisation par choc des ions dans un champ électrique intense. La méthode des scintillations a cet avantage qu'elle permet de distinguer sans ambiguïté la réception simultanée de deux particules, celles-ci n'arrivant jamais au même point de l'écran. En revanche de faibles scintillations peuvent passer inapercues. De plus, la méthode ne se prête pas à un enregistrement entièrement automatique et ne permet pas non plus de préciser l'instant exact de la réception. La méthode électrométrique permet, au contraire, un enregistrement extrêmement satisfaisant. L'expérience est montée de la manière suivante: l'image lumineuse, très fine, qui sert à repérer la position de l'électromètre, est obtenuc sur une fente derrière laquelle tourne un cylindre enregistreur, enveloppé de papier sensible; quand l'électromètre reste fixe, l'impression obtenue est,

1. Rutuenfond et Geigen, Phil. Mag., 20 (1910) 698-707. Note de M. Bateman. Mardsex et Bırate, Proc. Phys. Sor. of london, 23 (1911) 367-375. aprìs étalement du papier, une ligne droite trìs fine; chaque impulsion, subie par l'électromètre, se traduit sur l'impression par un crochet dont le point de départ est très nettement déterminé. Les graphiques acluellement obtenus sont d'une grande beauté; les détails relatifs à ce travail, scront publiés ultéricurement.

Les intervalles de temps qui séparent les inslants de réception ou d'émission des particules peurent être mesurés arec une grande précision sur les graphiques dont il vient d'ĉtre question, si le mourement du cylindre est uniforme et si l'on en connait la vitesse. J'ai fait ce travail pour une série de courbes comprenant 1080 intervalles et j’ai étudié comment varie avec le temps $t$ le nombre $n$ des intervalles compris cntre $t$ ct $t+0$, où $\theta$ représente un intervalle fixe, de grandeur convenable (aussi petit que possible, à condition que le nombre des intervalles qui interviennent reste encore suffisamment grand). J'ai trouvé que $n$ varie suivant une loi exponentielle, de sorte que l'on a $n=n_{0} e^{-\frac{t}{\tau}}$ ò̀ $\tau$ désigne l'interralle mojen et

$$
\frac{n_{0}}{\theta}=\frac{\mathrm{N}}{\tau}
$$

$\mathrm{N}$ étant le nombre total des intervalles.

Cette loi est la même que celle indiquée par MM. Marsden et Barratt. Elle est lout à fait de la mème forme que cel!e qui donne, pour un certain nombre de projectiles lancés dans un gaz, le nombre de ceux qui ne rencontreront une molécule de ce gaz qu'après avoir franchi une distance, comprise entre $x$ et $x+d x$. Le parcours moyen des projectiles joue ici le même ròle que l'intervalle moyen d'émission dans le problème qui nous occupe.

Ces expériences seront poursuivies arec un appareil enregistreur plus parfait au point de vue de la régulari!é du mouvement, et qui est actuellement en construction.

Il est utile de signaler l'application suivante qui a été faite en ce qui concerne les résultats indiqués. Quand on procède à la numération des particules $\alpha$ sur les graphiques obtenus à l'électromètre, on doit nécessairement laisser échapper un certain nombre de particules. Si, en effet, deux particules sont émises à un intervalle de temps trop court, l'électromitre qui possède toujours une certaine inerlie, ne pourra síparer les deux impulsions et n'enregistrera qu'un seul crochet. Il est vrai que ces impulsions qui correspondent à deux particules sont généralenent plus importantes, mais l'on ne saurait en tirer des conclusions fermes, car, même avec un appareil où toutes les particules devraient, semble-t-il, intervenir de la même manière, il n'a pas étí possible d'obtenir que les impulsions soient, en général, de grandeur tout à fait égale. (In peut remédier à cet inconvénient en étudiant la loi de distribution des intervalles et en utilisant cette loi pour calculer le nombre des particules correspondant 
à lintervalle d'émission qui constitue pour le dispositif utilisé l'intervalle limite accessible à l'observation.

Ce mode de correction a été appliqué avec avantage ì la numération des particules; la correction est facile à faire; elle ne nécessite que la connaissance de l'intervalle moyen.

[Manuscrit reçu le 27 Aon̂t 1911.] 